\title{
Non-data-aided ML SNR estimation for AWGN channels with deterministic interference
}

\author{
Fangjiong Chen, Yabing Kang, Hua Yu ${ }^{*}$ and Fei Ji
}

\begin{abstract}
Communication channels not only suffer from ambient noise but also from deterministic interference. In this paper, we consider signal-to-noise ratio (SNR) estimation in the presence of constant deterministic interference. A maximum likelihood (ML) non-data-aided algorithm is proposed for SNR estimation. We first consider a real-valued model and then extend this to a complex-valued model. The proposed algorithm applies an iterative approach initialized with approximate closed form estimates so as to guarantee stability and convergence. Furthermore, the Cramer-Rao bound (CRB) is also derived as the theoretical limit of the jitter variance. Computer simulations based on pulse-amplitude modulation (PAM) and quadrature amplitude modulation (QAM) sources show that the performance of the proposed algorithm is close to the CRB.
\end{abstract}

Keywords: SNR estimation; Maximum likelihood estimation; Non-data aided estimation; Cramer-Rao bound

\section{Introduction}

Besides ambient noise, the communication channels may suffer from deterministic interference. In terrestrial wireless systems, the competing users sharing the same spectrum resource, or simply the drift of the system's baseline, may introduce some sort of deterministic interference $[1,2]$. When a direct conversion receiver is applied, the demodulator output is usually impaired by a direct current (DC) offset due to self-mixing [3], which might be considered as some sort of deterministic interference. More recently, based on an experimental underwater communication system, Wang et al. [4] observed that unknown users transmitting multiple sonar waveforms in the same environment may lead to significant performance degradation. In [4], the deterministic interference is modelled as known waveform with unknown parameters. Interference reconstruction and cancelation was applied to improve the system performance.

The signal-to-noise ratio (SNR), defined as the ratio of the signal power to the noise power, is frequently used as the system performance measure [5-8]. In cases where interference is present, the interference can be estimated and removed from the estimated signal $[2,3]$. In $[1,6,7]$, the signal-to-interference-plus-noise ratio (SINR), instead of

*Correspondence: yuhua@scut.edu.cn

School of Electronic and Information Engineering, South China University of

Technology, Guangzhou 510640, China
SNR, is applied as the system measure. In [1], a non-dataaided (NDA) algorithm, based on fourth-order statistics, was proposed for SINR estimation, where the interference was modeled as a constant. In [6] and [7], SINR estimation in cellular systems is investigated, where the interference stems from competing users in other cells.

In $[6,7]$, the interference was modelled as zero-mean random variable. The algorithms indeed cannot deal with deterministic interference. The fourth-order statisticsbased algorithm in [1] requires a large quantity of samples (more than 1,000). It may not be effective to assume that the interference is constant during thousands of symbols. The maximum likelihood (ML) algorithm in [2] needs only tens of samples. However, the algorithm assumes no attenuation of the source signal and hence is not applicable to SNR estimation.

Our goal in this paper is to develop a NDA SNR estimation algorithm which provides satisfactory estimates with a small size of samples (e.g., tens of samples). We assume a slowly time-varying channel such that over the observation interval, the channel gain and the interference can be assumed to be constant. In natural, therefore, this is an additive noise channel model with attenuation factor and deterministic interference during the observation interval.

\section{Springer}

(c) 2014 Chen et al.; licensee Springer. This is an Open Access article distributed under the terms of the Creative Commons Attribution License (http://creativecommons.org/licenses/by/2.0), which permits unrestricted use, distribution, and reproduction in any medium, provided the original work is properly cited. 
In this paper, an iterative maximum likelihood (ML) algorithm is proposed for SNR estimation. A simple moment-based estimator is applied to initialize the ML algorithm. Simulation results show that the proposed algorithm converges within 10 iterations. We also derive the theoretical performance bound, i.e., the Cramer-Rao bound (CRB) as the performance benchmark. Computer simulations based on pulse-amplitude modulation (PAM) and quadrature amplitude modulation (QAM) sources show that the performance of the proposed algorithm with tens of samples is close to the CRB.

\section{Development of the iterative ML algorithm}

\subsection{Real-valued system with PAM signaling}

We first consider a real-valued system with PAM signaling. The received signal is given by

$$
x_{n}=A s_{n}+I+v_{n},
$$

where $s_{n}$ is the source signal and $v_{n}$ is the ambient noise. We assume that $v_{n}$ is zero-mean white Gaussian noise with variance $\sigma^{2}$. A stands for the channel gain/attenuation, and $I$ is the deterministic interference. We assume that $s_{n}$ is generated randomly by a $M$-ary PAM source with equiprobable constellation points at $\pm(2 m-1), m=$ $1,2, \cdots, M / 2$, with $M=2^{p}$ and $p \in \mathbb{N}$. Therefore, the average power of a $M$-ary PAM signal is given by $\mathcal{E}_{\mathrm{PAM}}^{M}=$ $\left(M^{2}-1\right) / 3$. As a result, the SNR of the received signal is defined as

$$
\gamma=\frac{A^{2} \mathcal{E}_{\mathrm{PAM}}^{M}}{\sigma^{2}}
$$

We note that in the open literature [1], the SINR, defined as $\gamma=\frac{A^{2} \mathcal{E}_{\mathrm{PAM}}^{M}}{I^{2}+\sigma^{2}}$ has also been applied as the performance measure. On top of that, cancellation of the DC offset is discussed in [3] and [9], where the SNR is used as the major figure of merit (it was argued that the DC offset has a less significant impact on the system performance than noise [3]). In the current paper, the constant deterministic interference (i.e. the DC-offset) was estimated and suppressed from the received signal, and then the SNR is applied as the performance measure. Our theoretical analysis further shows that varying the value of deterministic interference has no effect on the accuracy of parameter estimation (i.e. the estimation of $A, I$ and $\sigma^{2}$; see also Section 3). Hence, we argue that the SNR is a more proper performance measure in the presence of deterministic interference.

We shall separately estimate $\left\{A, I, \sigma^{2}\right\}$ and then use these estimates to calculate $\gamma$. Without loss of generality, $A$ is assumed to be a positive number in this paper. The PDF of $x_{n}$ is given by

$$
\begin{aligned}
f\left(x_{n}\right)= & \frac{1}{M \sqrt{2 \pi \sigma^{2}}} \sum_{m=1}^{M / 2}\left\{\exp \left(-\frac{\left(x_{n}-I-A(2 m-1)\right)^{2}}{2 \sigma^{2}}\right)\right. \\
& \left.+\exp \left(-\frac{\left(x_{n}-I+A(2 m-1)\right)^{2}}{2 \sigma^{2}}\right)\right\} \\
= & \frac{2}{M \sqrt{2 \pi \sigma^{2}}} \exp \left(-\frac{\left(x_{n}-I\right)^{2}}{2 \sigma^{2}}\right) \sum_{m=1}^{M / 2} \\
& \exp \left(-\frac{A^{2}(2 m-1)^{2}}{2 \sigma^{2}}\right) \cosh \left(\frac{A\left(x_{n}-I\right)(2 m-1)}{\sigma^{2}}\right) .
\end{aligned}
$$

Assume there are $N$ available samples, denoted as $\mathbf{x}=\left[x_{1}, x_{2}, \cdots, x_{N}\right]$. Since the transmitted symbols are assumed to be independent and identically distributed (i.i.d.) and the additive noise is white, then the corresponding received samples are independent and their joint probability density function (PDF) is simply the product of the PDF of each sample. Consequently, the log-likelihood function of $\mathbf{x}$ is given by

$$
\begin{aligned}
L(\mathbf{x})=\ln \prod_{n=1}^{N} f\left(x_{n}\right)= & N \ln \left(\frac{2}{M \sqrt{2 \pi \sigma^{2}}}\right)-\sum_{n=1}^{N} \frac{\left(x_{n}-I\right)^{2}}{2 \sigma^{2}} \\
& +\sum_{n=1}^{N} \ln \left(\sum_{m=1}^{M / 2} h_{m, n}^{c}\right)
\end{aligned}
$$

where $h_{m, n}^{c}$ is given as

$$
h_{m, n}^{c}=\exp \left(-\frac{A^{2}(2 m-1)^{2}}{2 \sigma^{2}}\right) \cosh \left(\frac{A\left(x_{n}-I\right)(2 m-1)}{\sigma^{2}}\right),
$$

By forcing the derivatives $\frac{\partial L(\mathbf{x})}{\partial A}, \frac{\partial L(\mathbf{x})}{\partial I}, \frac{\partial L(\mathbf{x})}{\partial \sigma^{2}}$ to zero, we obtain the subsequent relationships:

$$
\begin{aligned}
A & =\frac{\sum_{n=1}^{N}\left(x_{n}-I\right) H_{n}^{s}}{\sum_{n=1}^{N} H_{n}^{c}} \\
I & =\frac{1}{N} \sum_{n=1}^{N}\left(x_{n}-A H_{n}^{s}\right), \\
\sigma^{2} & =\frac{1}{N} \sum_{n=1}^{N}\left(\left(x_{n}-I\right)^{2}+A^{2} H_{n}^{c}-2 A\left(x_{n}-I\right) H_{n}^{s}\right),
\end{aligned}
$$

where

$$
\begin{aligned}
H_{n}^{c} & =\frac{\sum_{m=1}^{M / 2}\left((2 m-1)^{2} h_{m, n}^{c}\right)}{\sum_{m=1}^{M / 2} h_{m, n}^{c}}, \\
H_{n}^{s} & =\frac{\sum_{m=1}^{M / 2}\left((2 m-1) h_{m, n}^{s}\right)}{\sum_{m=1}^{M / 2} h_{m, n}^{c}} \\
h_{m, n}^{s} & =\exp \left(-\frac{A^{2}(2 m-1)^{2}}{2 \sigma^{2}}\right) \sinh \left(\frac{A\left(x_{n}-I\right)(2 m-1)}{\sigma^{2}}\right) .
\end{aligned}
$$

It is not an easy task to obtain closed form solutions for these equations. Hence, we propose an iterative approach, 
which has to be initialized properly. For this purpose, we consider the initialization based on the moments of the received signal.

By directly calculating the first-order sample raw moment of the received signal, we obtain the following initialization of $I$ :

$$
\hat{I}_{0}=\frac{1}{N} \sum_{n=1}^{N} x_{n}
$$

The second- and fourth-order central moments of the signal population are respectively given as,

$$
\begin{aligned}
\mu_{2} & =E_{s, v}\left\{\left(x_{n}-I\right)^{2}\right\}=E_{s, v}\left\{\left(A s_{n}+v_{n}\right)^{2}\right\} \\
& =A^{2} E\left\{s_{n}^{2}\right\}+E\left\{v_{n}^{2}\right\}=A^{2} v_{2}+\sigma^{2}, \\
\mu_{4} & =E_{s, v}\left\{\left(x_{n}-I\right)^{4}\right\}=E_{s, v}\left\{\left(A s_{n}+v_{n}\right)^{4}\right\} \\
& =A^{4} v_{4}+6 A^{2} v_{2} \sigma^{2}+3 \sigma^{4},
\end{aligned}
$$

where $v_{2}=E\left\{s_{n}^{2}\right\}=\mathcal{E}_{\text {PAM }}^{M}$ and $v_{4}=E\left\{s_{n}^{4}\right\}$ are the secondand fourth-order raw moments of the $M$-ary PAM signal $\left\{s_{n}\right\}$, respectively. And

$$
v_{4}=\frac{2}{M} \sum_{m=1}^{M / 2}(2 m-1)^{4}=\frac{1}{15}\left(3 M^{2}-7\right)\left(M^{2}-1\right) .
$$

According to (13) and (14), one has an equation for $A$ as

$$
3 \mu_{2}^{2}-\mu_{4}=A^{4}\left(3 v_{2}^{2}-v_{4}\right)
$$

Equating the population moments with the sample moments, one moment-based estimator for $A$ can be derived as

$$
\hat{A}_{0}=\left(\frac{3 \hat{\mu}_{2}^{2}-\hat{\mu}_{4}}{3 v_{2}^{2}-v_{4}}\right)^{1 / 4}
$$

where $\hat{\mu}_{2}$ and $\hat{\mu}_{4}$ are the second- and fourth-order sample central moments of the received signal and are given by, respectively,

$$
\hat{\mu}_{2}=\frac{1}{N} \sum_{n=1}^{N}\left(x_{n}-\hat{I}_{0}\right)^{2}, \quad \hat{\mu}_{4}=\frac{1}{N} \sum_{n=1}^{N}\left(x_{n}-\hat{I}_{0}\right)^{4} .
$$

When $\hat{I}_{0}$ and $\hat{A}_{0}$ are available, $\sigma^{2}$ can be estimated by exploiting the second-order central moment of the received signal, as follows:

$$
\hat{\sigma}_{0}^{2}=\frac{1}{N} \sum_{n=1}^{N}\left(x_{n}-\hat{I}_{0}\right)^{2}-\hat{A}_{0}^{2} \mathcal{E}_{\mathrm{PAM}}^{M} .
$$

As a result, the moment-based estimator for $\gamma$ can be expressed as

$$
\hat{\gamma}_{0}=\frac{\hat{A}_{0} \mathcal{E}_{\mathrm{PAM}}^{M}}{\hat{\sigma}_{0}^{2}}
$$

These moment-based estimators are only valid in the high SNR range. Unfortunately, they usually require a large quantity of samples to obtain accurate estimation, which is not realistic in practical time-varying systems. In order to obtain a more accurate estimation and speed up the convergence, we propose that the iterative method in (6) to (8) is initialized by the closed form estimates given by (12), (17) and (19). The procedure stops when a predefined maximum of iterations is achieved or when the error is lower than a specified value. The algorithm is outlined in Algorithm 1, where $K$ is a predefined maximal number of iterations, $\varepsilon$ is a predefined small constant, and the iterative error $\varepsilon_{k}$ is defined as

$$
\varepsilon_{k}=\left(\frac{\hat{\gamma}_{k}-\hat{\gamma}_{k-1}}{\hat{\gamma}_{k}}\right)^{2}, \quad k=1,2, \cdots
$$

\section{Algorithm 1 Iterative ML SNR estimation algorithm for PAM signaling}

\section{Initialization:}

Set $k=0$, and calculate the initial estimation of $I, A$ and $\sigma^{2}$, denoted as $\hat{I}_{0}, \hat{A}_{0}$ and $\hat{\sigma}_{0}^{2}$ based on (12), (17) and (19), respectively;

\section{Iteration:}

1: repeat

2: $\quad$ Calculate the $k$-th estimation of $h_{m, n}^{c}$ and $h_{m, n}^{s}$ by (5) and (11), respectively, with $A, I$, and $\sigma^{2}$ replaced by $\hat{A}_{k}, \hat{I}_{k}$ and $\hat{\sigma}_{k}^{2}$, respectively; denote the results as $\hat{h}_{m, n, k}^{c}$ and $\hat{h}_{m, n, k}^{s}$;

3: $\quad$ Calculate the $k$-th estimation of $H_{n}^{c}$ and $H_{n}^{s}$ by (9) and (10), respectively, with $h_{m, n}^{c}$ and $h_{m, n}^{s}$ replaced by $\hat{h}_{m, n, k}^{c}$ and $\hat{h}_{m, n, k}^{s}$, respectively; denote the results as $\hat{H}_{n, k}^{c}$ and $\hat{H}_{n, k}^{s}$;

4: $\quad k \leftarrow k+1$

5: $\quad$ Calculate the $k$-th estimation of $A, I$ and $\sigma^{2}$ by the equations (6), (7) and (8), respectively, with $A, I, \sigma^{2}$, $H_{n}^{s}$ and $H_{n}^{c}$ replaced by $\hat{A}_{k-1}, \hat{I}_{k-1}, \hat{\sigma}_{k-1}^{2}, \hat{H}_{n, k-1}^{s}$ and $\hat{H}_{n, k-1}^{c}$, respectively; denote the results as $\hat{A}_{k}, \hat{I}_{k}$ and $\hat{\sigma}_{k}^{2}$.

6: until $k=K$ or $\varepsilon_{k}<\varepsilon$.

\section{Output:}

$\hat{A}_{k}, \hat{I}_{k}, \hat{\sigma}_{k}^{2}$, and $\hat{\gamma}_{k}=\hat{A}_{k}^{2} \mathcal{E}_{\mathrm{PAM}}^{M} / \hat{\sigma}_{k}^{2}$ are the asymptotic ML estimates of the parameters $A, I, \sigma^{2}$ and $\gamma$, respectively. 


\subsection{Complex-valued system with QAM signaling}

Considering the complex-valued signal system, the signal model is revised as

$$
\begin{aligned}
r_{n} & =G s_{n}+I+w_{n} \\
& =(A+j B)\left(a_{n}+j b_{n}\right)+(C+j D)+\left(u_{n}+j v_{n}\right) \\
& =\left(A a_{n}-B b_{n}+C+u_{n}\right)+j\left(B a_{n}+A b_{n}+D+v_{n}\right) \\
& \triangleq x_{n}+j y_{n} .
\end{aligned}
$$

We assume $s_{n}=a_{n}+j b_{n}$ is a $M$-ary square QAM source, that is, $s_{n} \in \mathcal{S}=\left\{S_{i q}=a_{i}+j b_{q}=\left(2 i-1-2^{p}\right)+j(2 q-1-\right.$ $\left.2^{p}\right), i, q=1, \cdots, 2^{p}$, where $M=2^{2 p}$ for any natural number $p, G=A+j B$ is the complex channel gain, $I=C+j D$ and $w_{n}=u_{n}+j v_{n}$ are respectively the complex interference and complex noise. The average power of the square QAM constellation power is given by $\mathcal{E}_{\mathrm{QAM}}^{M}=E\left\{\left|S_{i q}\right|^{2}\right\}=2(M-1) / 3$.

The SNR of the received signal is defined as

$$
\gamma=\frac{|G|^{2} \mathcal{E}_{\mathrm{QAM}}^{M}}{2 \sigma^{2}}=\frac{\left(A^{2}+B^{2}\right) \mathcal{E}_{\mathrm{QAM}}^{M}}{2 \sigma^{2}} .
$$

We shall separately estimate the unknown parameters of the vector $\left\{A, B, C, D, \sigma^{2}\right\}$ and then use these estimates to calculate $\gamma$. Without loss of generality, $A$ and $B$ are assumed to be positive numbers in this paper.

The joint PDF of the real and image parts of $r_{n}$ is given by:

$$
\begin{aligned}
f_{r_{n}}\left(x_{n}, y_{n}\right)= & \frac{1}{2 M \pi \sigma^{2}} \sum_{S_{i q} \in \mathcal{S}} \exp \left\{-\frac{\left(\left(x_{n}-C\right)-\left(A a_{i}-B b_{q}\right)\right)^{2}+\left(\left(y_{n}-D\right)-\left(B a_{i}+A b_{q}\right)\right)^{2}}{2 \sigma^{2}}\right\} \\
= & \frac{1}{2 M \pi \sigma^{2}} \exp \left\{-\frac{\left(x_{n}-C\right)^{2}+\left(y_{n}-D\right)^{2}}{2 \sigma^{2}}\right\} \\
& \sum_{S_{i q} \in \mathcal{S}} \exp \left\{-\frac{\left|G S_{i q}\right|^{2}}{2 \sigma^{2}}\right\} \exp \left\{\frac{\left(x_{n}-C\right)\left(A a_{i}-B b_{q}\right)+\left(y_{n}-D\right)\left(B a_{i}+A b_{q}\right)}{\sigma^{2}}\right\}
\end{aligned}
$$

According to the symmetry of the square QAM constellation, $f_{r_{n}}\left(x_{n}, y_{n}\right)$ can be written as

$$
\begin{aligned}
f_{r_{n}}\left(x_{n}, y_{n}\right)= & \frac{1}{2 M \pi \sigma^{2}} \exp \left\{-\frac{\left(x_{n}-C\right)^{2}+\left(y_{n}-D\right)^{2}}{2 \sigma^{2}}\right\} \sum_{S_{i q} \in \mathcal{S}} \exp \left\{-\frac{\left|G S_{i q}\right|^{2}}{2 \sigma^{2}}\right\} \\
& \exp \left\{\frac{\left(A\left(x_{n}-C\right)+B\left(y_{n}-D\right)\right) a_{i}+\left(A\left(y_{n}-D\right)-B\left(x_{n}-C\right)\right) b_{q}}{\sigma^{2}}\right\} \\
= & \frac{2}{M \pi \sigma^{2}} \exp \left\{-\frac{\left(x_{n}-C\right)^{2}+\left(y_{n}-D\right)^{2}}{2 \sigma^{2}}\right\} \\
& \sum_{i=1}^{2^{p-1}} \exp \left\{-\frac{\left(A^{2}+B^{2}\right)(2 i-1)^{2}}{2 \sigma^{2}}\right\} \cosh \left\{\frac{\left(A\left(x_{n}-C\right)+B\left(y_{n}-D\right)\right)(2 i-1)}{\sigma^{2}}\right\} \\
& \sum_{q=1}^{2^{p-1}} \exp \left\{-\frac{\left(A^{2}+B^{2}\right)(2 q-1)^{2}}{2 \sigma^{2}}\right\} \cosh \left\{\frac{\left(A\left(y_{n}-D\right)-B\left(x_{n}-C\right)\right)(2 q-1)}{\sigma^{2}}\right\} \\
= & \frac{2}{M \pi \sigma^{2}} \exp \left\{-\frac{\left(x_{n}-C\right)^{2}+\left(y_{n}-D\right)^{2}}{2 \sigma^{2}}\right\} H\left(X_{n}\right) H\left(Y_{n}\right)
\end{aligned}
$$


where

$$
\begin{aligned}
X_{n} & =A\left(x_{n}-C\right)+B\left(y_{n}-D\right), \\
Y_{n} & =A\left(y_{n}-D\right)-B\left(x_{n}-C\right), \\
H(Z) & =\sum_{i=1}^{2^{p-1}} h_{i}^{c}(Z), \\
h_{i}^{c}(Z) & =\exp \left\{-\frac{\left(A^{2}+B^{2}\right)(2 i-1)^{2}}{2 \sigma^{2}}\right\} \cosh \left(\frac{Z(2 i-1)}{\sigma^{2}}\right) .
\end{aligned}
$$

Assume there are $N$ available received samples, denoted as $\mathbf{r}=\left[r_{1}, r_{2}, \cdots, r_{N}\right]$. As a result, the logarithmic likelihood function, define as $L(\mathbf{r})=\ln \left(f_{r_{1}}(\cdot) f_{r_{2}}(\cdot) \cdots f_{r_{N}}(\cdot)\right)$ is given by

$$
\begin{aligned}
L(\mathbf{r})= & N \ln \left(\frac{2}{M \pi \sigma^{2}}\right)-\sum_{n=1}^{N} \frac{\left(x_{n}-C\right)^{2}+\left(y_{n}-D\right)^{2}}{2 \sigma^{2}} \\
& +\sum_{n=1}^{N} \ln \left(H\left(X_{n}\right)\right)+\sum_{n=1}^{N} \ln \left(H\left(Y_{n}\right)\right)
\end{aligned}
$$

By forcing the derivatives $\frac{\partial L(\mathbf{r})}{\partial A}, \frac{\partial L(\mathbf{r})}{\partial B}, \frac{\partial L(\mathbf{r})}{\partial C}, \frac{\partial L(\mathbf{r})}{\partial D}, \frac{\partial L(\mathbf{r})}{\partial \sigma^{2}}$ (see Appendix) to zero, we obtain the following equations:

$$
\begin{aligned}
A= & \frac{1}{\mathcal{H}} \sum_{n=1}^{N}\left(\left(x_{n}-C\right) H_{s}\left(X_{n}\right)+\left(y_{n}-D\right) H_{s}\left(Y_{n}\right)\right), \\
B= & \frac{1}{\mathcal{H}} \sum_{n=1}^{N}\left(\left(y_{n}-D\right) H_{s}\left(X_{n}\right)-\left(x_{n}-C\right) H_{s}\left(Y_{n}\right)\right), \\
C= & \frac{1}{N} \sum_{n=1}^{N} x_{n}-\frac{1}{N} \sum_{n=1}^{N}\left(A H_{s}\left(X_{n}\right)-B H_{s}\left(Y_{n}\right)\right), \\
D= & \frac{1}{N} \sum_{n=1}^{N} y_{n}-\frac{1}{N} \sum_{n=1}^{N}\left(B H_{s}\left(X_{n}\right)+A H_{s}\left(Y_{n}\right)\right), \\
\sigma^{2}= & \frac{1}{2 N}\left\{\left(A^{2}+B^{2}\right) \mathcal{H}+\sum_{n=1}^{N}\left(\left(x_{n}-C\right)^{2}+\left(y_{n}-D\right)^{2}\right)\right. \\
& \left.-2 \sum_{n=1}^{N}\left(X_{n} H_{s}\left(X_{n}\right)+Y_{n} H_{s}\left(Y_{n}\right)\right)\right\}
\end{aligned}
$$

where

$$
\begin{aligned}
\mathcal{H} & =\sum_{n=1}^{N}\left(H_{c}\left(X_{n}\right)+H_{c}\left(Y_{n}\right)\right), \\
H_{c}(Z) & =\frac{1}{H(Z)} \sum_{i=1}^{2^{p-1}}(2 i-1)^{2} h_{i}^{c}(Z), \\
H_{s}(Z) & =\frac{1}{H(Z)} \sum_{i=1}^{2^{p-1}}(2 i-1) h_{i}^{s}(Z), \\
h_{i}^{s}(Z) & =\exp \left\{-\frac{\left(A^{2}+B^{2}\right)(2 i-1)^{2}}{2 \sigma^{2}}\right\} \sinh \left(\frac{Z(2 i-1)}{\sigma^{2}}\right) .
\end{aligned}
$$

Similar to the case of PAM signaling, we adopt the moment-based estimators as initialization and then apply iterative estimation based on (31) to (35) to refine the estimated results. At first, the moment estimate of $I$ is given as

$$
\hat{I}_{0}=\hat{C}_{0}+j \hat{D}_{0}=\frac{1}{N} \sum_{n=1}^{N} r_{n}=\frac{1}{N} \sum_{n=1}^{N} x_{n}+j \frac{1}{N} \sum_{n=1}^{N} y_{n}
$$

The second- and fourth-order central moments of the signal's real and imaginary parts are respectively given as,

$$
\begin{aligned}
\mu_{x^{2}} & =E_{s, w}\left\{\left(x_{n}-C\right)^{2}\right\}=E_{s, u}\left\{\left(A a_{n}-B b_{n}+u_{n}\right)^{2}\right\} \\
& =\left(A^{2}+B^{2}\right) v_{2}+\sigma^{2}, \\
\mu_{y^{2}} & =E_{s, w}\left\{\left(y_{n}-D\right)^{2}\right\}=E_{s, v}\left\{\left(B a_{n}+A b_{n}+v_{n}\right)^{2}\right\} \\
& =\left(A^{2}+B^{2}\right) v_{2}+\sigma^{2}=\mu_{x^{2}}, \\
\mu_{x^{4}} & =E_{s, w}\left\{\left(x_{n}-C\right)^{4}\right\}=E_{s, u}\left\{\left(A a_{n}-B b_{n}+u_{n}\right)^{4}\right\} \\
& =\left(A^{4}+B^{4}\right) v_{4}+6 A^{2} B^{2} v_{2}^{2}+6\left(A^{2}+B^{2}\right) v_{2} \sigma^{2}+3 \sigma^{4},
\end{aligned}
$$

$$
\begin{aligned}
\mu_{y^{4}}= & E_{s, w}\left\{\left(y_{n}-D\right)^{4}\right\}=E_{s, v}\left\{\left(B a_{n}+A b_{n}+v_{n}\right)^{4}\right\} \\
= & \left(A^{4}+B^{4}\right) v_{4}+6 A^{2} B^{2} v_{2}^{2}+6\left(A^{2}+B^{2}\right) v_{2} \sigma^{2} \\
& +3 \sigma^{4}=\mu_{x^{4}}, \\
\mu_{x^{2} y^{2}}= & E_{s, w}\left\{\left(x_{n}-C\right)^{2}\left(y_{n}-D\right)^{2}\right\} \\
= & E_{s, w}\left\{\left(A a_{n}-B b_{n}+u_{n}\right)^{2}\left(B a_{n}+A b_{n}+v_{n}\right)^{2}\right\} \\
= & 2 A^{2} B^{2} v_{4}+\left(A^{4}+B^{4}-4 A^{2} B^{2}\right) v_{2}^{2} \\
& +2\left(A^{2}+B^{2}\right) v_{2} \sigma^{2}+\sigma^{4},
\end{aligned}
$$

where $v_{2}=E\left\{a_{n}^{2}\right\}=E\left\{b_{n}^{2}\right\}=\mathcal{E}_{\mathrm{OAM}}^{M} / 2=(M-1) / 3$ and $v_{4}=E\left\{a_{n}^{4}\right\}=E\left\{b_{n}^{4}\right\}$ are the second- and fourthorder raw moments of the real/imaginary part of the $M$ ary QAM signal $\left\{s_{n}\right\}$, respectively. And

$$
v_{4}=\frac{2}{\sqrt{M}} \sum_{i=1}^{\sqrt{M} / 2}(2 i-1)^{4}=\frac{1}{15}(3 M-7)(M-1) .
$$

According to (41) to (45), one has two equations for $|G|^{2}=$ $A^{2}+B^{2}$ as

$$
\begin{aligned}
& 4 \mu_{x^{2}}^{2}-\mu_{x^{4}}-\mu_{x^{2} y^{2}}=\left(A^{2}+B^{2}\right)^{2}\left(3 v_{2}^{2}-v_{4}\right), \\
& 4 \mu_{y^{2}}^{2}-\mu_{y^{4}}-\mu_{x^{2} y^{2}}=\left(A^{2}+B^{2}\right)^{2}\left(3 v_{2}^{2}-v_{4}\right) .
\end{aligned}
$$

Equating the population moments with the sample moments, one moment-based estimator for $|G|^{2}=A^{2}+$ $B^{2}$ can be derived as

$$
\left|\hat{G}_{0}\right|^{2}=\sqrt{\frac{4 \hat{\mu}_{x^{2}}^{2}+4 \hat{\mu}_{y^{2}}^{2}-\hat{\mu}_{x^{4}}-\hat{\mu}_{y^{4}}-2 \hat{\mu}_{x^{2} y^{2}}}{2\left(3 v_{2}^{2}-v_{4}\right)}},
$$

where $\hat{\mu}_{x^{2}}, \hat{\mu}_{y^{2}}, \hat{\mu}_{x^{4}}, \hat{\mu}_{y^{4}}$ and $\hat{\mu}_{x^{2} y^{2}}$ are the secondand fourth-order sample central moments of the 
real/imaginary part of received signal, and are given by, respectively,

$$
\begin{aligned}
& \hat{\mu}_{x^{2}}=\frac{1}{N} \sum_{n=1}^{N}\left(x_{n}-\hat{C}_{0}\right)^{2}, \quad \hat{\mu}_{y^{2}}=\frac{1}{N} \sum_{n=1}^{N}\left(y_{n}-\hat{D}_{0}\right)^{2} \\
& \hat{\mu}_{x^{4}}=\frac{1}{N} \sum_{n=1}^{N}\left(x_{n}-\hat{C}_{0}\right)^{4}, \quad \hat{\mu}_{y^{4}}=\frac{1}{N} \sum_{n=1}^{N}\left(y_{n}-\hat{D}_{0}\right)^{4} \\
& \hat{\mu}_{x^{2} y^{2}}=\frac{1}{N} \sum_{n=1}^{N}\left(x_{n}-\hat{C}_{0}\right)^{2}\left(y_{n}-\hat{D}_{0}\right)^{2}
\end{aligned}
$$

When $\hat{I}_{0}$ and $\hat{G}_{0}$ are available, $\sigma^{2}$ can be estimated by exploiting the second-order central moment of the received signal, as follows:

$$
\hat{\sigma}_{0}^{2}=\frac{1}{2 N} \sum_{n=1}^{N}\left|r_{n}-\hat{I}_{0}\right|^{2}-\frac{1}{2}\left|\hat{G}_{0}\right|^{2} \mathcal{E}_{\mathrm{QAM}}^{M} .
$$

As a result, the moment-based estimator for $\gamma$ can be expressed as

$$
\hat{\gamma}_{0}=\frac{\left|\hat{G}_{0}\right|^{2} \mathcal{E}_{\mathrm{QAM}}^{M}}{\hat{\sigma}_{0}^{2}}
$$

It can be seen from (41) to (45) that $A$ and $B$ are asymmetric such that they cannot be decoupled from these equations. Therefore, they are temporally assumed to be equal and then they are refined by the iteration. That is, the rough estimates of $A$ and $B$ are given as

$$
\hat{A}_{0}=\hat{B}_{0}=\sqrt{\left|\hat{G}_{0}\right|^{2} / 2} \text {. }
$$

The iterative algorithm is similar to the case of PAM signaling and will not be shown here for saving space.

\section{The Cramer-Rao bound}

\subsection{The real-valued system with PAM signaling}

In this section, we derive the CRB for unbiased estimation of $\gamma$. Let $\boldsymbol{\theta}=\left[\theta_{1}, \theta_{2}\right]=\left[A, \sigma^{2}\right]$ and $\gamma=g(\boldsymbol{\theta})=$ $A^{2} \mathcal{E}_{\mathrm{PAM}}^{M} / \sigma^{2}$. The CRB of SNR is given by [8], i.e.,

$$
\mathrm{CRB}(\gamma)=\frac{\partial \mathrm{g}(\boldsymbol{\theta})}{\partial \boldsymbol{\theta}} \mathbf{F}^{-1}\left(\frac{\partial \mathrm{g}(\boldsymbol{\theta})}{\partial \boldsymbol{\theta}}\right)^{\mathrm{T}}
$$

where

$$
\frac{\partial g(\boldsymbol{\theta})}{\partial \boldsymbol{\theta}}=\left[\frac{\partial g(\boldsymbol{\theta})}{\partial A}, \frac{\partial g(\boldsymbol{\theta})}{\partial \sigma^{2}}\right]=\left[\frac{2 A}{\sigma^{2}},-\frac{A^{2}}{\sigma^{4}}\right] \mathcal{E}_{\mathrm{PAM}}^{M},
$$

F is the Fisher information matrix (FIM) defined as

$$
\mathbf{F}_{\ell_{J}}=E\left\{\frac{\partial L(\mathbf{x})}{\partial \theta_{\ell}} \frac{\partial L(\mathbf{x})}{\partial \theta_{J}}\right\}, \quad \ell, J=1,2,
$$

where $E\{\cdot\}$ denotes the expectation w.r.t the random variables $\left\{s_{n}\right\}$ and $\left\{v_{n}\right\}$. After some algebra, we have

$$
\begin{aligned}
\frac{\partial L(\mathbf{x})}{\partial \theta_{\ell}}= & \frac{1}{\sigma^{2}} \sum_{n=1}^{N} \phi_{\ell}\left(x_{n}\right), \quad \ell=1,2, \\
\phi_{1}\left(x_{n}\right)= & \left(x_{n}-I\right) H_{n}^{s}-A H_{n}^{c}=\left(A s_{n}+v_{n}\right) H_{n}^{s}-A H_{n}^{c} \\
\phi_{2}\left(x_{n}\right)= & \frac{1}{2 \sigma^{2}}\left(\left(x_{n}-I\right)^{2}+A^{2} H_{n}^{c}-2 A\left(x_{n}-I\right) H_{n}^{s}\right)-\frac{1}{2} \\
= & \frac{1}{2 \sigma^{2}}\left(\left(A s_{n}+v_{n}\right)^{2}+A^{2} H_{n}^{c}-2 A\left(A s_{n}+v_{n}\right) H_{n}^{s}\right) \\
& -\frac{1}{2} .
\end{aligned}
$$

Note that $x_{n}-I$ has been replaced by $A s_{n}+v_{n}$ such that $\phi_{\ell}\left(x_{n}\right)$ has no relation to $I$, as can be verified by (60) and (61). By using this kind of substitution, it is easily observed that $H_{n}^{c}$ and $H_{n}^{s}$ do not include $I$ as well. For convenience, we will denote $\phi_{\ell}\left(x_{n}\right)$ as $\phi_{\ell}\left(s_{n}, v_{n}\right)$ so that $\mathbf{F}_{\ell J}$ can be calculated as

$$
\begin{aligned}
\mathbf{F}_{\ell_{J}}= & \frac{1}{\sigma^{4}} E\left\{\sum_{n=1}^{N} \sum_{n^{\prime}=1}^{N} \phi_{J}\left(s_{n}, v_{n}\right) \phi_{\ell}\left(s_{n^{\prime}}, v_{n^{\prime}}\right)\right\}, \quad \ell, J=1,2, \\
= & \frac{1}{\sigma^{4}} \sum_{n=1}^{N} E_{s, v}\left\{\phi_{\ell}\left(s_{n}, v_{n}\right) \phi_{J}\left(s_{n}, v_{n}\right)\right\} \\
& +\frac{1}{\sigma^{4}} \sum_{n=1}^{N} \sum_{n^{\prime}=1, n^{\prime} \neq n}^{N} E_{s, v}\left\{\phi_{\ell}\left(s_{n^{\prime}}, v_{n^{\prime}}\right)\right\} E_{s, v}\left\{\phi_{J}\left(s_{n}, v_{n}\right)\right\} \\
= & \frac{N}{\sigma^{4}} E_{s}\left\{\eta_{\ell_{J}}(s)\right\}+\frac{N(N-1)}{\sigma^{4}} E_{s}\left\{\mu_{\ell}(s)\right\} E_{s}\left\{\mu_{J}(s)\right\} \quad \text { (62) }
\end{aligned}
$$

where

$E_{s}\left\{\eta_{\ell_{J}}(s)\right\}=\frac{1}{M} \sum_{m=1}^{M} \eta_{\ell_{J}}(2 m-1-M), \quad \ell, j=1,2$,

$E_{s}\left\{\mu_{\ell}(s)\right\}=\frac{1}{M} \sum_{m=1}^{M} \mu_{\ell}(2 m-1-M), \quad \ell=1,2$,

$$
\begin{array}{ll}
\eta_{\ell_{J}}(s)=\int_{-\infty}^{\infty} \phi_{\ell}(s, v) \phi_{J}(s, v) p(v) d v, & \ell, J=1,2, \\
\mu_{\ell}(s)=\int_{-\infty}^{\infty} \phi_{\ell}(s, v) p(v) d v, & \ell=1,2,
\end{array}
$$

and $p(v)=\frac{1}{\sigma \sqrt{2 \pi}} \exp \left(-v^{2} / 2 \sigma^{2}\right)$ is the PDF of the additive white Gaussian noise $v$ with variance $\sigma^{2}$. It is not possible to obtain closed-form expressions for the above integrations. Numerical computation is applied to calculate $\eta_{\ell J}(s), \mu_{\ell}(s)$ and then the CRB. Note that the reciprocals of the diagonal elements of $\mathbf{F}$ provide the CRB for the estimation of $\left\{A, \sigma^{2}\right\}$. It is interesting to remark that the CRBs of $\left\{A, \sigma^{2}, \gamma\right\}$ are not related to the value of $I$, which means 
that, in theory, an increase of the interference level would not hurt the accuracy in estimating $\left\{A, \sigma^{2}, \gamma\right\}$.

\subsection{The complex-valued system with QAM signaling}

Next, we consider the complex-valued system with QAM Signaling. Let $\boldsymbol{\theta}=\left[\theta_{1}, \theta_{2}, \theta_{3}\right]=\left\{A, B, \sigma^{2}\right\}$ and $\gamma=g(\boldsymbol{\theta})=$ $\frac{\left(A^{2}+B^{2}\right) \mathcal{E}_{\mathrm{QAM}}^{M}}{2 \sigma^{2}}$. The CRB of SNR is given by [8]

$$
\operatorname{CRB}(\gamma)=\frac{\partial g(\boldsymbol{\theta})}{\partial \boldsymbol{\theta}} \mathbf{F}^{-1}\left(\frac{\partial \mathrm{g}(\boldsymbol{\theta})}{\partial \boldsymbol{\theta}}\right)^{\mathrm{T}}
$$

$\frac{\partial g(\boldsymbol{\theta})}{\partial \boldsymbol{\theta}}=\left[\frac{\partial g(\boldsymbol{\theta})}{\partial A}, \frac{\partial g(\boldsymbol{\theta})}{\partial B}, \frac{\partial g(\boldsymbol{\theta})}{\partial \sigma^{\mathbf{2}}}\right]=\frac{\mathcal{E}_{\mathrm{QAM}}^{M}}{\sigma^{2}}\left[A, B,-\frac{A^{2}+B^{2}}{2 \sigma^{2}}\right]$.

$\mathbf{F}$ is the Fisher information matrix, whose elements are defined as

$$
\mathbf{F}_{\ell_{J}}=E\left\{\frac{\partial L(\mathbf{r})}{\partial \theta_{\ell}} \frac{\partial L(\mathbf{r})}{\partial \theta_{J}}\right\}, \quad \ell, J=1,2,3,
$$

where $E\{\cdot\}$ denotes the expectation w.r.t to the random variables $\left\{a_{n}\right\},\left\{b_{n}\right\},\left\{u_{n}\right\}$, and $\left\{v_{n}\right\}$.

With some calculation, we have (see Appendix)

$$
\begin{aligned}
\frac{\partial L(\mathbf{r})}{\partial \theta_{\ell}}= & \frac{1}{\sigma^{2}} \sum_{n=1}^{N} \phi_{\ell}\left(r_{n}\right), \quad \ell=1,2,3, \\
\phi_{1}\left(r_{n}\right)= & \left(\left(x_{n}-C\right) H_{s}\left(X_{n}\right)+\left(y_{n}-D\right) H_{s}\left(Y_{n}\right)\right) \\
& -A\left(H_{c}\left(X_{n}\right)+H_{c}\left(Y_{n}\right)\right), \\
\phi_{2}\left(r_{n}\right)= & \left(\left(y_{n}-D\right) H_{s}\left(X_{n}\right)-\left(x_{n}-C\right) H_{s}\left(Y_{n}\right)\right)-B\left(H_{c}\left(X_{n}\right)\right. \\
& \left.+H_{c}\left(Y_{n}\right)\right), \\
\phi_{3}\left(r_{n}\right)= & -1+\frac{1}{2 \sigma^{2}}\left\{\left(x_{n}-C\right)^{2}+\left(y_{n}-D\right)^{2}+\left(A^{2}+B^{2}\right)\left(H_{c}\left(X_{n}\right)\right.\right. \\
& \left.\left.+H_{c}\left(Y_{n}\right)\right)-2\left(X_{n} H_{s}\left(X_{n}\right)+Y_{n} H_{s}\left(Y_{n}\right)\right)\right\} .
\end{aligned}
$$

In fact, since

$$
\begin{aligned}
x_{n}-C & =A a_{n}-B b_{n}+u_{n}, \\
y_{n}-D & =B a_{n}+A b_{n}+v_{n}, \\
X_{n} & =\left(A^{2}+B^{2}\right) a_{n}+A u_{n}+B v_{n}, \\
Y_{n} & =\left(A^{2}+B^{2}\right) b_{n}+A v_{n}-B u_{n},
\end{aligned}
$$

then $H_{c}\left(X_{n}\right), H_{c}\left(Y_{n}\right), H_{s}\left(X_{n}\right)$, and $H_{s}\left(Y_{n}\right)$ are unrelated to the values of the interference level $C$ and $D$, so are $\left\{\phi_{\ell}\left(r_{n}\right)\right\}$. Therefore, $\phi_{\ell}\left(r_{n}\right)$ can be rewritten as $\phi_{\ell}\left(s_{n}, w_{n}\right)$, and $\mathbf{F}_{\ell_{J}}$ can be calculated by

$$
\begin{aligned}
\mathbf{F}_{\ell_{J}}= & \frac{1}{\sigma^{4}} E\left\{\sum_{n=1}^{N} \sum_{n^{\prime}=1}^{N} \phi_{J}\left(s_{n}, w_{n}\right) \phi_{\ell}\left(s_{n^{\prime}}, w_{n^{\prime}}\right)\right\}, \quad \ell, J=1,2,3, \\
= & \frac{1}{\sigma^{4}} \sum_{n=1}^{N} E_{s, w}\left\{\phi_{\ell}\left(s_{n}, w_{n}\right) \phi_{J}\left(s_{n}, w_{n}\right)\right\} \\
& +\frac{1}{\sigma^{4}} \sum_{n=1}^{N} \sum_{n^{\prime}=1, n^{\prime} \neq n}^{N} E_{s, w}\left\{\phi_{\ell}\left(s_{n^{\prime}}, w_{n^{\prime}}\right)\right\} E_{s, w}\left\{\phi_{J}\left(s_{n}, w_{n}\right)\right\} \\
= & \frac{N}{\sigma^{4}} E_{s}\left\{\eta_{\ell_{J}}(s)\right\}+\frac{N(N-1)}{\sigma^{4}} E_{s}\left\{\mu_{\ell}(s)\right\} E_{s}\left\{\mu_{J}(s)\right\}
\end{aligned}
$$

where

$$
\begin{aligned}
E_{s}\left\{\eta_{\ell_{J}}(s)\right\} & =\frac{1}{M} \sum_{i=1}^{2^{p}} \sum_{q=1}^{2^{p}} \eta_{\ell_{J}}\left(S_{i q}\right), & \ell, J=1,2,3, \\
E_{s}\left\{\mu_{\ell}(s)\right\} & =\frac{1}{M} \sum_{i=1}^{2^{p}} \sum_{q=1}^{2^{p}} \mu_{\ell}\left(S_{i q}\right), & \ell=1,2,3, \\
\eta_{\ell_{J}}(s) & =\int_{-\infty}^{\infty} \int_{-\infty}^{\infty} \phi_{\ell}(s, w) \phi_{J}(s, w) p_{w}(u, v) d u d v, & \ell, J=1,2,3, \\
\mu_{\ell}(s) & =\int_{-\infty}^{\infty} \int_{-\infty}^{\infty} \phi_{\ell}(s, w) p_{w}(u, v) d u d v, & \ell=1,2,3,
\end{aligned}
$$

and $p_{w}(u, v)=\frac{1}{2 \pi \sigma^{2}} \exp \left(-\frac{u^{2}+v^{2}}{2 \sigma^{2}}\right)$ is the joint PDF of the real and image parts of the complex additive white Gaussian noise $w=u+j v$ with variance $\sigma^{2}$. In the simulation, numerical computation is applied to calculate $\eta_{\ell_{J}}(s)$, $\mu_{\ell}(s)$ and then the CRB.

\section{Numerical results}

Computer simulation results are presented to show the performance of the proposed algorithm. Unless otherwise specified, a sample size of $N=80,2 \times 10^{4}$ independent trials and an interference-to-noise ratio (INR) of $0 \mathrm{~dB}$ are applied in the simulation. To guarantee convergence, $\epsilon$ is set to a small value of $10^{-6}$ and $K$ is set to a large value of 100 . To obtain a performance measure for a fair comparison of different parameters, we use the normalized sample mean square error (NMSE) defined by

$$
\mathrm{NMSE}=\frac{1}{N_{t r}} \sum_{i=1}^{N_{t r}}\left(\frac{\hat{\gamma}_{i}-\gamma}{\gamma}\right)^{2},
$$

where $N_{t r}$ is the number of total independent trials and $\hat{\gamma}_{i}$ is the estimated value of the true SNR $\gamma$ from the $i$ th trial.

Figure 1 shows the convergence property of the proposed ML estimator (MLE). It can be observed that the algorithm has fast convergence speed since that it converges in several iterations, and the performance of the proposed MLE is close to the CRB and significantly outperforms the moment-based estimator $(k=0)$.

Figure 2 shows the estimation performance under different sample sizes. It can be observed that the proposed ML estimator results in almost identical performance to the CRB. We therefore argue that tens of samples is enough to obtain efficient estimation. Although in this paper we assume time-invariant interference level and noise power, the proposed algorithm indeed is applicable to slow timevarying systems where the parameters can be assumed to be invariant within tens of samples. We also observe that an increased number of samples will be required to obtain an accurate SNR estimation, especially at low SNR.

The estimation performance of PAM and QAM signaling with different modulation orders is shown in Figure 3. 


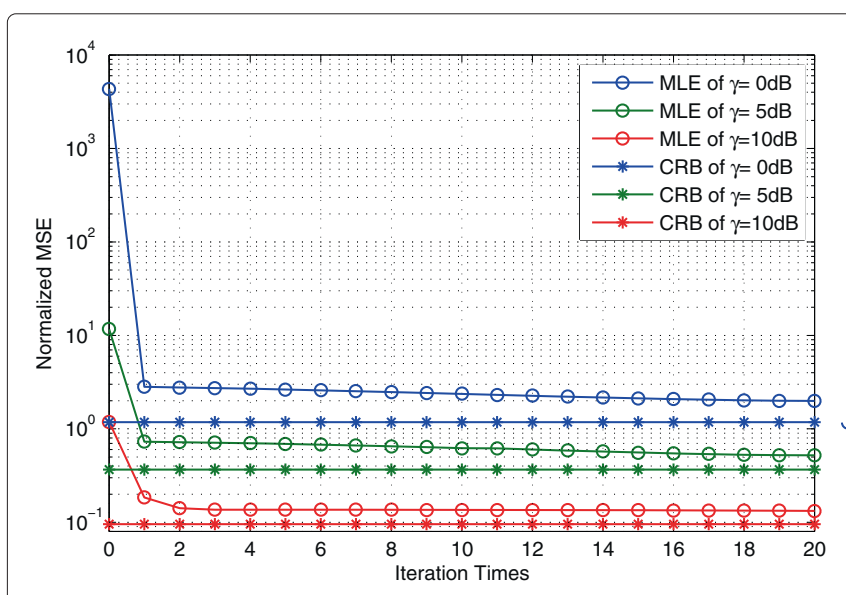

(a) 4PAM

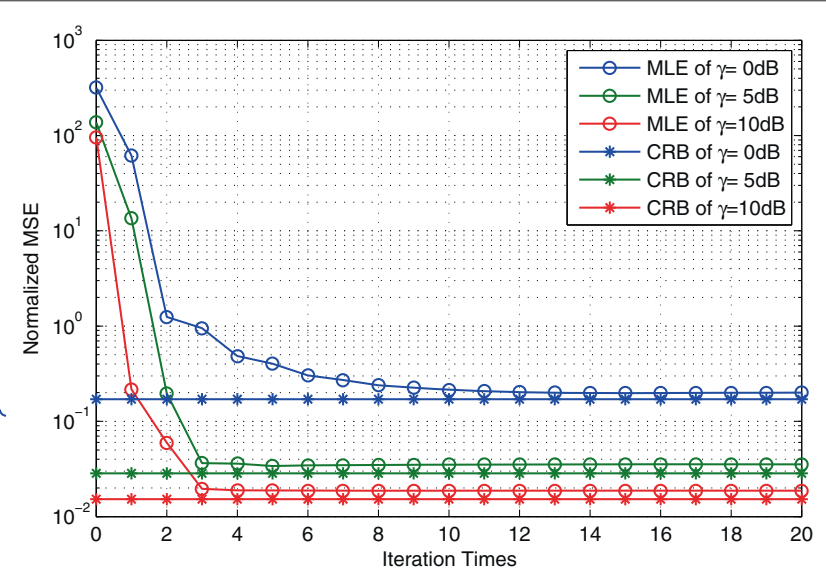

(b) QPSK

Figure 1 Estimation performance in terms of the iteration times $\boldsymbol{k}$. (a) 4PAM and (b) QPSK.

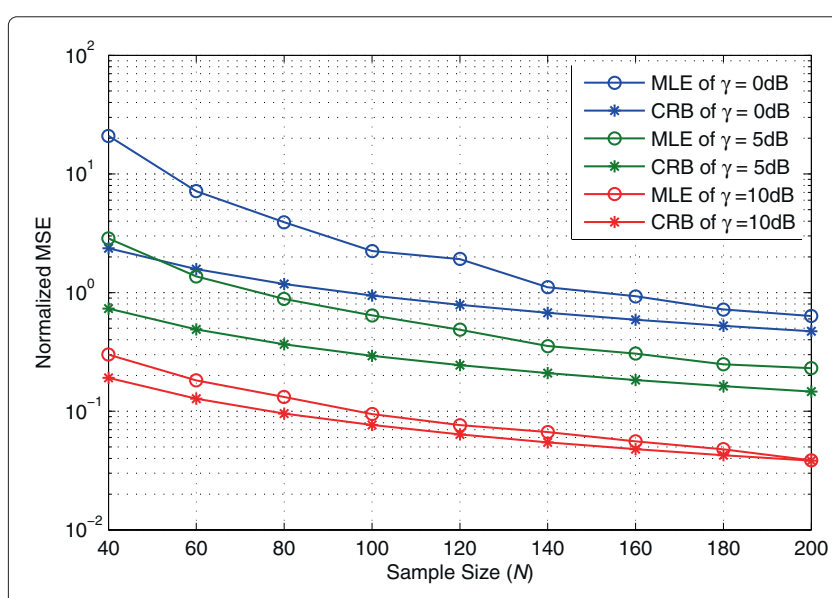

(a) PAM

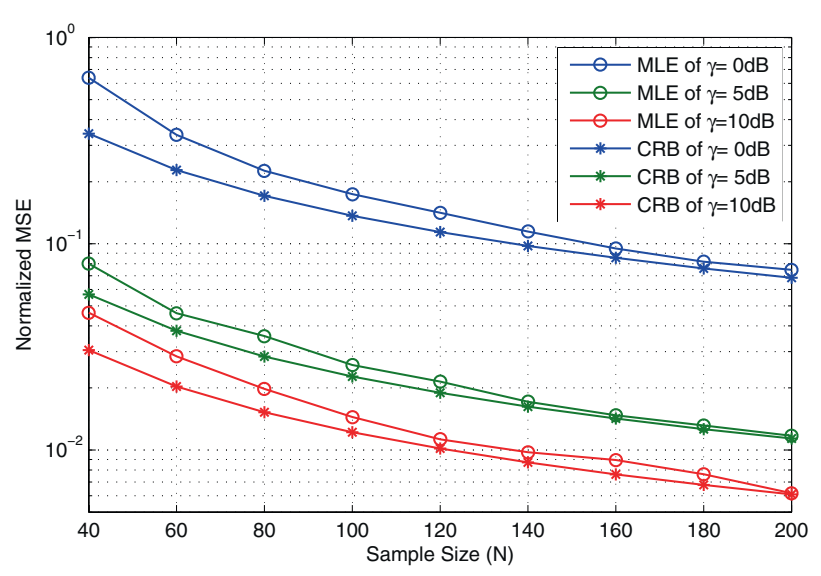

(b) QPSK

Figure 2 Estimation performance in terms of the sample size $\boldsymbol{N}$. (a) 4PAM and (b) QPSK.

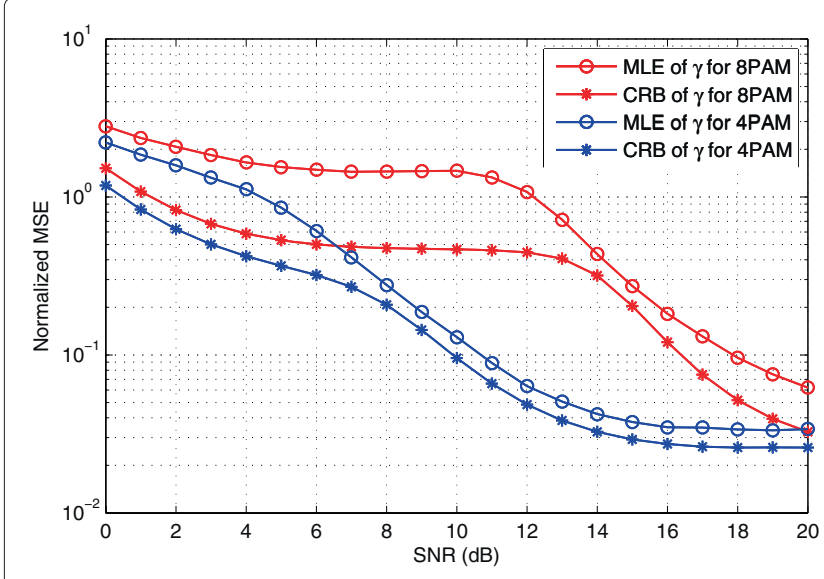

(a) M-PAM

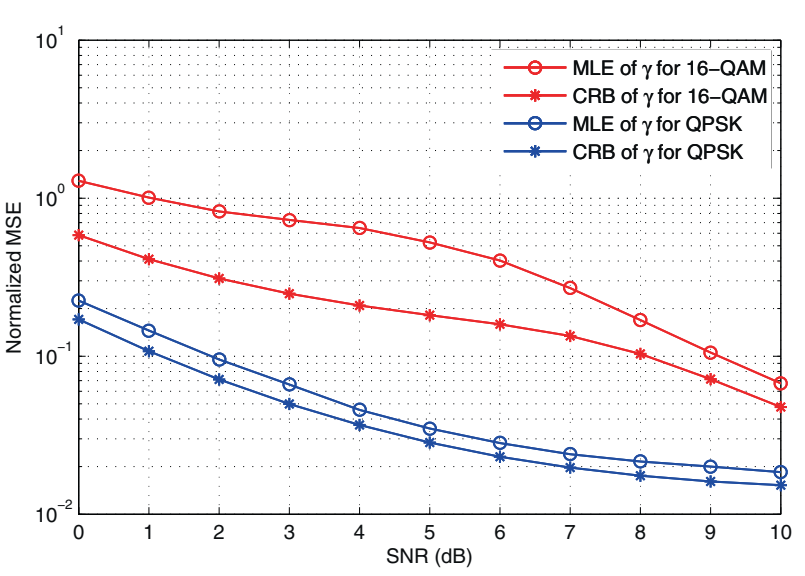

(b) M-QAM

Figure 3 Estimation performance comparison between different modulation orders. (a) M-PAM and (b) M-QAM. 


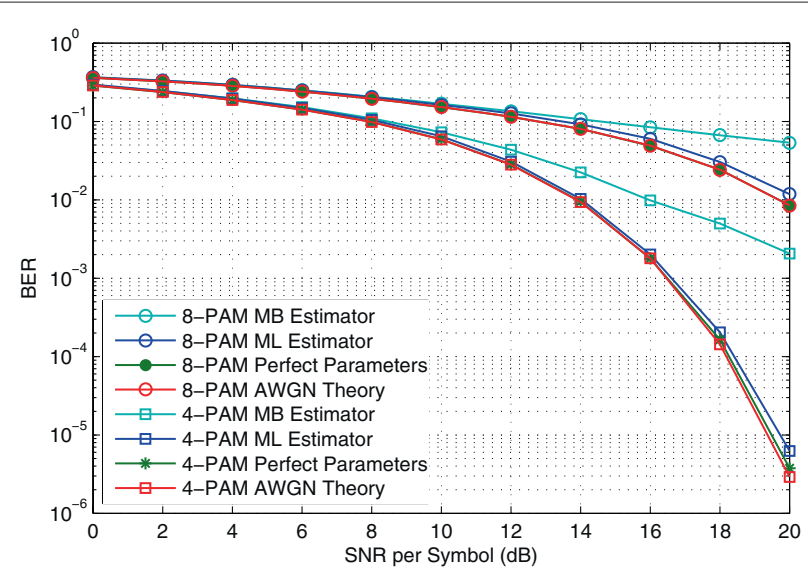

(a) M-PAM

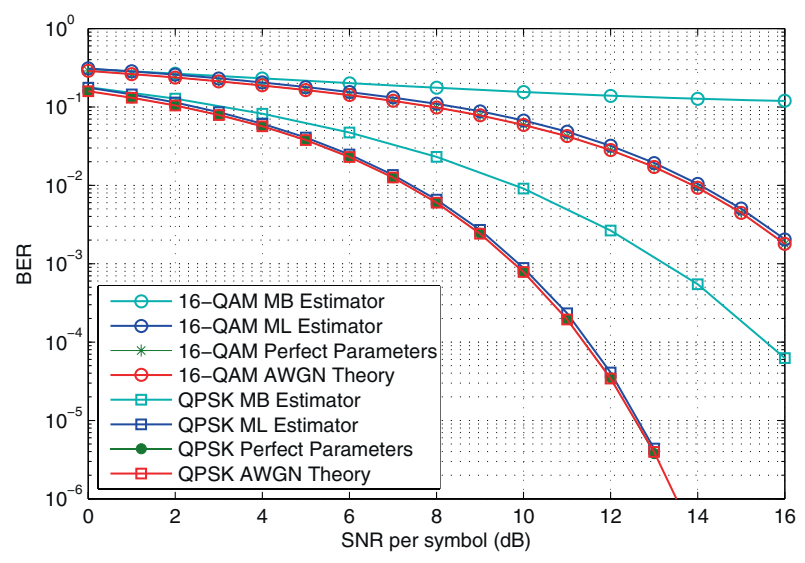

(b) M-QAM

Figure 4 BER performance comparison. (a) M-PAM and (b) M-QAM.

The figure suggests that both the ML and MB estimators work better for smaller constellation sizes. It can be observed from the above figures that the NMSE of the proposed MLE for $\gamma$ is decreasing as the SNR increases. Some numerical evaluation and simulation results, which are not shown here because of limited space, reveal that the variation of interference level has no effect on the NMSE of MLE and CRB of channel parameters of $\left\{G, I, \sigma^{2}, \gamma\right\}$. This result agrees also with the analysis in Section 3 .

Figure 4 shows the bit-error-rate (BER) performance of the proposed algorithm. We apply the estimated channel gain and interference level for symbol detection. As a comparison, the moment-based estimator is also evaluated in the simulation. It can be observed that the proposed estimator significantly outperforms the moment-based estimator in the cases of high SNR, and it shows almost the same performance as knowing the perfect system parameters. On the other side, it also can be seen from Figure 3 and Figure 4 that the large estimation deviation of the system parameters at low SNR has little effect on the BER performance.

\section{Conclusions}

SNR estimation in an additive noise channel with attenuation factor and deterministic interference has been considered. A non-data-aided iterative estimation algorithm has been proposed based on the ML criterion. The proposed algorithm provides satisfactory performance, although this is achieved to the price of an increased computational complexity. Simulation results show that the algorithm provides applicable results on the order of tens of samples. And, its performance is almost identical to the corresponding bounds over a wide range of the true SNR. Moreover, the additive noise channel of this paper also includes the case where the interference is determined by a Gaussian random variable with non-zero mean. In this case, the interference can be divided into a constant and a zero-mean Gaussian random variable. As far as the SNR is concerned, the zero-mean Gaussian random part of the interference can be attributed to the additive Gaussian noise. Therefore, the model is also validated in this case. This case represents channels where there are many weak interferers plus noise but no dominant interferers and also represents channels where there is one dominant constant interferer plus noise. Furthermore, next step of work may focus on extending the algorithm to multipath channel environments.

\section{Appendix}

Derivations of the likelihood function for QAM signaling

$$
\begin{aligned}
\frac{\partial L(\mathbf{r})}{\partial A}= & \sum_{n=1}^{N} \frac{1}{H\left(X_{n}\right)} \sum_{i=1}^{2^{p-1}}\left\{\frac{(2 i-1)\left(x_{n}-C\right)}{\sigma^{2}} h_{i}^{s}\left(X_{n}\right)-\frac{(2 i-1)^{2} A}{\sigma^{2}} h_{i}^{c}\left(X_{n}\right)\right\} \\
& +\sum_{n=1}^{N} \frac{1}{H\left(Y_{n}\right)} \sum_{i=1}^{2^{p-1}}\left\{\frac{(2 i-1)\left(y_{n}-D\right)}{\sigma^{2}} h_{i}^{s}\left(Y_{n}\right)-\frac{(2 i-1)^{2} A}{\sigma^{2}} h_{i}^{c}\left(Y_{n}\right)\right\} \\
= & \frac{1}{\sigma^{2}} \sum_{n=1}^{N}\left(\left(x_{n}-C\right) H_{s}\left(X_{n}\right)+\left(y_{n}-D\right) H_{s}\left(Y_{n}\right)\right) \\
& -\frac{A}{\sigma^{2}} \sum_{n=1}^{N}\left(H_{c}\left(X_{n}\right)+H_{c}\left(Y_{n}\right)\right) \\
\frac{\partial L(\mathbf{r})}{\partial B}= & \sum_{n=1}^{N} \frac{1}{H\left(X_{n}\right)} \sum_{i=1}^{2^{p-1}}\left\{\frac{(2 i-1)\left(y_{n}-D\right)}{\sigma^{2}} h_{i}^{s}\left(X_{n}\right)-\frac{(2 i-1)^{2} B}{\sigma^{2}} h_{i}^{c}\left(X_{n}\right)\right\} \\
& -\sum_{n=1}^{N} \frac{1}{H\left(Y_{n}\right)} \sum_{i=1}^{2^{p-1}}\left\{\frac{(2 i-1)\left(x_{n}-C\right)}{\sigma^{2}} h_{i}^{s}\left(Y_{n}\right)-\frac{(2 i-1)^{2} B}{\sigma^{2}} h_{i}^{c}\left(Y_{n}\right)\right\} \\
= & \frac{1}{\sigma^{2}} \sum_{n=1}^{N}\left(\left(y_{n}-D\right) H_{s}\left(X_{n}\right)-\left(x_{n}-C\right) H_{s}\left(Y_{n}\right)\right) \\
& -\frac{B}{\sigma^{2}} \sum_{n=1}^{N}\left(H_{c}\left(X_{n}\right)+H_{c}\left(Y_{n}\right)\right)
\end{aligned}
$$




$$
\begin{aligned}
\frac{\partial L(\mathbf{r})}{\partial C}= & \sum_{n=1}^{N} \frac{x_{n}-C}{\sigma^{2}}-\sum_{n=1}^{N} \frac{1}{H\left(X_{n}\right)} \sum_{i=1}^{2^{p-1}} \frac{(2 i-1) A}{\sigma^{2}} h_{i}^{s}\left(X_{n}\right)+\sum_{n=1}^{N} \frac{1}{H\left(Y_{n}\right)} \\
& \times \sum_{i=1}^{2^{p-1}} \frac{(2 i-1) B}{\sigma^{2}} h_{i}^{s}\left(Y_{n}\right) \\
= & \frac{1}{\sigma^{2}} \sum_{n=1}^{N}\left(x_{n}-C\right)-\frac{A}{\sigma^{2}} \sum_{n=1}^{N} H_{s}\left(X_{n}\right)+\frac{B}{\sigma^{2}} \sum_{n=1}^{N} H_{s}\left(Y_{n}\right) \\
\frac{\partial L(\mathbf{r})}{\partial D}= & \sum_{n=1}^{N} \frac{y_{n}-D}{\sigma^{2}}-\sum_{n=1}^{N} \frac{1}{H\left(X_{n}\right)} \sum_{i=1}^{2^{p-1}} \frac{(2 i-1) B}{\sigma^{2}} h_{i}^{s}\left(X_{n}\right)-\sum_{n=1}^{N} \frac{1}{H\left(Y_{n}\right)} \\
& \times \sum_{i=1}^{2^{p-1}} \frac{(2 i-1) A}{\sigma^{2}} h_{i}^{s}\left(Y_{n}\right) \\
= & \frac{1}{\sigma^{2}} \sum_{n=1}^{N}\left(y_{n}-D\right)-\frac{B}{\sigma^{2}} \sum_{n=1}^{N} H_{s}\left(X_{n}\right)-\frac{A}{\sigma^{2}} \sum_{n=1}^{N} H_{s}\left(Y_{n}\right) \\
\frac{\partial L(\mathbf{r})}{\partial \sigma^{2}}= & -\frac{N}{\sigma^{2}}+\sum_{n=1}^{N} \frac{\left(x_{n}-C\right)^{2}+\left(y_{n}-D\right)^{2}}{2 \sigma^{4}} \\
& +\sum_{n=1}^{N} \frac{1}{H\left(X_{n}\right)} \sum_{i=1}^{2^{p-1}}\left\{\frac{\left(A^{2}+B^{2}\right)(2 i-1)^{2}}{2 \sigma^{4}} h_{i}^{c}\left(X_{n}\right)-\frac{(2 i-1) X_{n}}{\sigma^{4}} h_{i}^{s}\left(X_{n}\right)\right\} \\
& +\sum_{n=1}^{N} \frac{1}{\sigma^{4}} \sum_{n=1} \sum_{n} \sum_{n}^{p-1}\left\{\frac{\left(X^{2}+B_{n}\right)(2 i-1)^{2}}{2 \sigma^{4}} h_{i}^{c}\left(Y_{n}\right)-\frac{(2 i-1) Y_{n}}{\sigma^{4}} h_{i}^{s}\left(Y_{n}\right)\right\} \\
= & \left.\frac{N}{\sigma^{2}}+\frac{1}{2 \sigma^{4}}\left(A^{2}+B^{2}\right) \mathcal{H}+\frac{1}{2 \sigma^{4}} \sum_{n=1}^{N}\left(\left(x_{n}-C\right)^{2}+\left(y_{n}-D\right)^{2}\right)\right) \\
& \\
& \\
&
\end{aligned}
$$

\section{Competing interests}

The authors declare that they have no competing interests.

\section{Acknowledgements}

The authors would like to thank the anonymous reviewers for their very contributive comments in making the paper more appealing. This work is supported in part by the National Natural Science Foundation of China (61372081, 61171083, 61271209), Key Grant Project of Chinese Ministry of Education (313021), Program for New Century Excellent Talents in University (NCET-12-0196) and the Fundamental Research Funds for the Central Universities of SCUT (2014ZG0028, 2014ZG0042).

\section{Received: 27 March 2013 Accepted: 6 March 2014}

Published: 25 March 2014

\section{References}

1. Y Chen, NC Beaulieu, NDA estimation of SINR for QAM signals. IEEE Commun. Lett. 9(8), 688-690 (2005)

2. $\mathrm{F}$ Chen, $\mathrm{F} \mathrm{Ji}, \mathrm{T}$ Cao, $\mathrm{S}$ Xiong, Maximum likelihood based measurement of interference level and noise power for memoryless gaussian channel with deterministic interference. IEEE Trans. Commun. 60(1), 19-22 (2012)

3. CH Yih, Analysis and compensation of DC offset in OFDM systems over frequency-selective rayleigh fading channels. IEEE Trans Vehicular Technol. 58(7), 3436-3446 (2009)

4. Z Wang, S Zhou, J Catipovic, P Willett, Parameterized cancellation of partial-band partial-block-duration interference for underwater acoustic OFDM. IEEE Trans. Signal Process. 60(4), 1782-1795 (2012)

5. N Merhav, Universal decoding for memoryless Gaussian channels with a deterministic interference. IEEE Trans. Inform. Theory 39(4), 1261-1269 (1993)

6. Y Zhao, J Wu, S Lu, Efficient SINR estimating with accuracy control in large scale cognitive radio networks, in 2011 IEEE 17th International Conference on Parallel and Distributed Systems (ICPADS), (LOS Alamitos, CA, USA: IEEE Computer Society, 2011), pp. 549-556
7. S Sorooshyari, CW Tan, HV Poor, On maximum-likelihood SINR estimation of MPSK in a multiuser fading channel. IEEE Trans. Vehicular Technol. 59(8), 4175-4181 (2010)

8. W Gappmair, Cramer-Rao lower bound for non-data-aided SNR estimation of linear modulation schemes. IEEE Trans. Commun. 56(5), 689-693 (2008)

9. M Inamori, AM Bostamam, Y Sanada, H Minami, IQ imbalance compensation scheme in the presence of frequency offset and dynamic DC offset for a direct conversion receiver. IEEE Trans. Wireless Commun. 8(5), 2214-2220 (2009)

\section{doi:10.1186/1687-1499-2014-45}

Cite this article as: Chen et al: Non-data-aided ML SNR estimation for AWGN channels with deterministic interference. EURASIP Journal on Wireless Communications and Networking 2014 2014:45.

\section{Submit your manuscript to a SpringerOpen ${ }^{\mathcal{O}}$ journal and benefit from:}

- Convenient online submission

Rigorous peer review

- Immediate publication on acceptance

- Open access: articles freely available online

- High visibility within the field

- Retaining the copyright to your article

Submit your next manuscript at $\gg$ springeropen.com 